\title{
Cargo Scheduling Decision Support for Offshore Oil and Gas Production: A Case Study
}

\author{
Elham Mardaneh • Qun Lin • Ryan Loxton • Nicola Wilson
}

Received: date / Accepted: date

\begin{abstract}
Woodside Energy Ltd (Woodside), Australia's largest independent oil and gas company, operates multiple oil and gas facilities off the coast of Western Australia. These facilities require regular cargo shipments from supply vessels based in Karratha, Western Australia. In this paper, we describe a decision support model for scheduling the cargo shipments to minimize travel cost and trip duration, subject to various operational restrictions including vessel capacities, cargo demands at the facilities, time windows at the facilities, and base opening times. The model is a type of non-standard vehicle routing problem involving multiple supply vessels — a primary supply vessel that visits every facility during a round trip taking at most one week, and other supply vessels that are used on an ad hoc basis when the primary vessel cannot meet all cargo demands. We validate the model via test simulations using real data provided by Woodside.
\end{abstract}

E. Mardaneh

Department of Mathematics and Statistics, Curtin University, Perth, Australia

E-mail: elham.mardaneh@curtin.edu.au

Q. Lin

Department of Mathematics and Statistics, Curtin University, Perth, Australia

E-mail: q.lin@curtin.edu.au

R. Loxton

Department of Mathematics and Statistics, Curtin University, Perth, Australia

E-mail: r.loxton@curtin.edu.au

N. Wilson

Woodside Energy Ltd, Perth, Australia

E-mail: nicola.wilson@woodside.com.au 
Keywords Cargo scheduling · Vehicle routing problem · Mixed-integer linear programming · Oil and gas production

Mathematics Subject Classification (2000) 90B06 $\cdot 90 \mathrm{~B} 50 \cdot 90 \mathrm{~B} 90 \cdot 90 \mathrm{C} 11$

\section{Introduction}

This paper describes the results of a joint research project between Curtin University, Australia and Woodside Energy Ltd (Woodside), Australia's largest independent oil and gas company. The purpose of the project was to develop a decision support model for optimally scheduling the cargo deliveries at Woodside-operated offshore oil and gas facilities in the Indian Ocean off the coast of North West Australia.

The cargo deliveries are performed by a vessel fleet consisting of one primary supply vessel and multiple secondary vessels. In the past, the fleet operated a "taxi-style" service whereby cargo deliveries were routed on an ad hoc basis according to requests from the offshore facilities. The work in this paper was commissioned by Woodside to investigate a fixed schedule format whereby the primary supply vessel performs the same round trip each week, visiting every offshore facility once according to fixed arrival times. This fixed schedule format (which may require chartering a larger primary vessel in the future) is cheaper to implement and allows the offshore facilities to plan their operations with more certainty regarding cargo deliveries.

The purpose of this paper is to present a decision support model for designing the new schedule format. The design problem involves determining an optimal seven-day schedule during which the primary supply vessel visits every facility on a single round trip starting and finishing at the supply base. The secondary supply vessels are used on an ad hoc basis to fill any cargo delivery shortfalls left over by the primary vessel. The problem is to choose the trip start times and vessel routes (which are defined by the facility visit sequence and facility arrival times) to ensure that prescribed cargo delivery requirements are satisfied. The optimization objective incorporates two criteria: travel cost (less travel is better) and trip duration (less time is better). There is a natural trade-off between these two criteria; for example, the minimum-distance schedule typically requires long periods of unproductive waiting. The proposed decision support model provides a tool for finding the best compromise between these competing objectives.

The cargo scheduling problem described above can be viewed as a vehicle routing problem with side constraints

[4,6]. These constraints include the following:

1. Cargo carried by a vessel cannot exceed the vessel's capacity; 
2. Each offshore facility has a certain cargo demand that must be delivered;

3. The supply base is only open for part of each day and servicing can be paused during base closure;

4. The offshore facilities may be open for all or part of each day;

5. There is a fixed helicopter schedule for transporting personnel and, due to limited manpower at the offshore facilities, cargo operations cannot occur while a facility is being prepared for helicopter arrival;

6. Night cargo operations are prohibited at some offshore facilities;

7. Each vessel's tour cannot exceed seven days in duration; and

8. A minimum amount of cargo must be offloaded on each visit.

Constraints 4-6 above define a set of disjoint arrival windows for each offshore facility: a vessel must arrive during one of these windows to ensure that its cargo offload operation does not intersect with facility closure, helicopter arrival preparation, or possibly night hours if there are restrictions on night-time loading. Thus, our cargo scheduling problem can be viewed as a vehicle routing problem with time windows, a class of problems that has been extensively studied in the literature [4]. In addition, our problem also includes the non-standard side constraints 3, 7, and 8. These non-standard constraints have been considered individually in the literature; see, for example, references $[1,3,7]$ for minimum delivery constraints and references $[2,5]$ for trip duration constraints. However, we are unaware of any previous work that incorporates all of constraints 1-8 above into a combined optimization model.

The model described in this paper, which has been designed specifically for Woodside's cargo scheduling problem, does indeed contain all of constraints 1-8. Since the model is challenging to solve for realistic industry scenarios, we present a heuristic method for determining an initial feasible schedule, which can be used to initialize (and ultimately accelerate the convergence of) commercial optimization solvers such as CPLEX. The proposed model has been applied at a strategic level to advise Woodside on fleet replacement options and different schedule formats.

\section{Model Description}

We consider a network $(\mathcal{N}, \mathcal{A})$, where $\mathcal{N}$ is the node set (containing nodes representing the facilities) and $\mathcal{A}$ is the arc set (containing arcs representing the transportation links between facilities). In the node set $\mathcal{N}$, the supply base is represented by a single node 0 and each offshore facility is represented by multiple nodes, one for each arrival window. Let $\mathcal{F}$ denote the set of offshore facilities and let $\mathcal{N}_{f}$ denote the set of nodes corresponding to facility 
$f \in \mathcal{F}$ (each node in $\mathcal{N}_{f}$ corresponds to a different time window for facility $f$ ). Furthermore, let $\mathcal{K}=\left\{k_{P}\right\} \cup \mathcal{K}_{S}$ denote the set of vessels, where $k_{P}$ represents the primary supply vessel (conducts a closed tour visiting every facility) and the elements of $\mathcal{K}_{S}$ represent the secondary supply vessels (used on an ad hoc basis when needed).

Vessel $k \in \mathcal{K}$ is defined by the following parameters: $Q^{k}$ (deck-space capacity in $\left.\mathrm{m}^{2}\right), \tau_{i j}^{k}$ (travel time required to traverse $(i, j) \in \mathcal{A}$ ), $\alpha_{i j}^{k}$ (fixed cost coefficient corresponding to $(i, j) \in \mathcal{A}$ ), and $\beta_{i j}^{k}$ (variable cost coefficient corresponding to $(i, j) \in \mathcal{A})$. The cost coefficients define the travel costs for the optimization problem; vessel $k$ traversing arc $(i, j)$ incurs a fixed cost of $\alpha_{i j}^{k}$ and a variable cost of $\beta_{i j}^{k}$ for every $\mathrm{m}^{2}$ of cargo flow. In practice, these coefficients are typically chosen to measure travel distance or fuel consumption. For each offshore facility $f \in \mathcal{F}$, the key parameters are: $q_{f}$ (cargo demand in $\mathrm{m}^{2}$ of deck-space), $\delta_{f}^{k}$ (service duration for vessel $k \in \mathcal{K}$ ), and $\epsilon_{f}^{k}$ (minimum cargo offload in $\mathrm{m}^{2}$ for vessel $k \in \mathcal{K}$ ).

Our model is based on the following assumptions: time is measured in hours; the vessel fleet is heterogeneous; service interruptions due to facility closure are allowed at the base but not at offshore facilities; each vessel undergoes an initial base service before departing from the base; the open/close times of the supply base are the same on each day; and vessels start servicing facilities immediately after arrival. Since service interruptions are not allowed at offshore facilities, a vessel arriving at an offshore facility during a certain working shift must complete the service during the same shift. In contrast, servicing at the base can be paused while the base is closed.

The primary supply vessel performs a single closed tour visiting every offshore facility; each secondary supply vessel performs at most one closed tour and is not required to visit all facilities. The primary supply vessel's tour takes at most one week (inclusive of base service). All other tours must be completed within the same time frame as the primary vessel's tour. Since each tour can begin on any day of the week and may take as long as 7 days, we consider the problem over an indicative 14-day time horizon.

Let $\delta_{0}^{k}$ denote the service duration at the base for vessel $k \in \mathcal{K}$. Furthermore, let $\left[a_{0}^{d}, b_{0}^{d}\right]$ denote the supply base's opening period on day $d \in\{1, \ldots, 14\}$, and let $\left[a_{i}, b_{i}\right]$ denote the arrival time window for node $i \in \mathcal{N} \backslash\{0\}$. Since the base's open and close times are the same on each day, $a_{0}^{d+1}=a_{0}^{d}+24$ and $b_{0}^{d+1}=b_{0}^{d}+24$ for each $d \in\{1, \ldots, 13\}$. Furthermore,

$$
b_{0}^{1}-a_{0}^{1}=b_{0}^{2}-a_{0}^{2}=\cdots=b_{0}^{14}-a_{0}^{14} .
$$

The decision variables in the model are defined below:

- $x_{i j}^{k}=$ binary variable indicating whether vessel $k \in \mathcal{K}$ traverses $\operatorname{link}(i, j) \in \mathcal{A}\left(x_{i j}^{k}=1\right.$ if this occurs; $x_{i j}^{k}=0$ otherwise) 
- $z_{d l}^{k}=$ binary variable indicating whether vessel $k \in \mathcal{K}$ starts the initial base service on day $d$ and completes the base service on day $d+l \in\{d, d+1, \ldots, 14\}$

- $y_{i j}^{k}=$ cargo flow transported by vessel $k \in \mathcal{K}$ along link $(i, j) \in \mathcal{A}$

- $s_{i}^{k}=$ arrival time of vessel $k \in \mathcal{K}$ at node $i \in \mathcal{N} \backslash\{0\}$

- $s_{0}^{k}=$ base service start time of vessel $k \in \mathcal{K}$

- $t^{k}=$ trip duration of vessel $k \in \mathcal{K}$

We define $s_{i}^{k}=0$ if vessel $k$ never visits node $i$. Similarly, for any secondary supply vessel $k \in \mathcal{K}_{S}$, we define $s_{0}^{k}=0$ and $t^{k}=0$ if vessel $k$ never leaves the base (recall that the primary supply vessel must leave the base, but there is no such requirement for the secondary vessels).

The performance metrics of interest are travel cost and trip duration. These quantities are defined as follows:

$$
\text { Travel Cost Function }=\sum_{k \in \mathcal{K}} \sum_{(i, j) \in \mathcal{A}} \alpha_{i j}^{k} x_{i j}^{k}+\sum_{k \in \mathcal{K}} \sum_{(i, j) \in \mathcal{A}} \beta_{i j}^{k} y_{i j}^{k}
$$

and

$$
\text { Trip Duration Function }=\sum_{k \in \mathcal{K}} t^{k} .
$$

In the proposed decision support model, a composite objective function is formed by taking the linear combination (with appropriate weights) of the travel cost and trip duration functions. This composite objective function should be minimized subject to the constraints listed below (in what follows, $M$ is a sufficiently large positive number).

- Bound constraints on the cargo flows:

$$
0 \leq y_{i j}^{k} \leq Q^{k}, \quad(i, j) \in \mathcal{A}, \quad k \in \mathcal{K} .
$$

- Bound constraints on the facility arrival times:

$$
\sum_{j \in \mathcal{N}} a_{i} x_{j i}^{k} \leq s_{i}^{k} \leq \sum_{j \in \mathcal{N}} b_{i} x_{j i}^{k}, \quad i \in \mathcal{N}_{f}, \quad f \in \mathcal{F}, \quad k \in \mathcal{K} .
$$

- Bound constraints on the base service start times for the primary vessel:

$$
\sum_{d=1}^{7} \sum_{l=0}^{7} a_{0}^{d} z_{d l}^{k_{P}} \leq s_{0}^{k_{P}} \leq \sum_{d=1}^{7} \sum_{l=0}^{7} b_{0}^{d} z_{d l}^{k_{P}}
$$

- Bound constraints on the base service start times for each secondary vessel:

$$
\sum_{d=1}^{14} \sum_{l=0}^{14-d} a_{0}^{d} z_{d l}^{k} \leq s_{0}^{k} \leq \sum_{d=1}^{14} \sum_{l=0}^{14-d} b_{0}^{d} z_{d l}^{k}, \quad k \in \mathcal{K}_{S}
$$


- Bound constraints on the trip durations:

$$
0 \leq t^{k} \leq \sum_{j \in \mathcal{N}} 168 x_{0 j}^{k}, \quad k \in \mathcal{K}
$$

- Zero commodity flow if the corresponding link is not traversed:

$$
y_{i j}^{k} \leq Q^{k} x_{i j}^{k}, \quad(i, j) \in \mathcal{A}, \quad k \in \mathcal{K}
$$

- Cargo flow cannot exceed deck-space capacity:

$$
\sum_{j \in \mathcal{N}} y_{0 j}^{k} \leq Q^{k}, \quad k \in \mathcal{K}
$$

- Each offshore facility is visited precisely once by the primary supply vessel:

$$
\sum_{j \in \mathcal{N}} \sum_{i \in \mathcal{N}_{f}} x_{j i}^{k_{P}}=1, \quad f \in \mathcal{F}
$$

- Each offshore facility is visited at most once by each secondary supply vessel:

$$
\sum_{j \in \mathcal{N}} \sum_{i \in \mathcal{N}_{f}} x_{j i}^{k} \leq 1, \quad f \in \mathcal{F}, \quad k \in \mathcal{K}_{S}
$$

- Conservation of vessel flow at each node:

$$
\sum_{j \in \mathcal{N}} x_{j i}^{k}=\sum_{j \in \mathcal{N}} x_{i j}^{k}, \quad i \in \mathcal{N}, \quad k \in \mathcal{K}
$$

- Primary supply vessel performs a single closed tour:

$$
\sum_{j \in \mathcal{N}} x_{0 j}^{k_{P}}=1
$$

- Each secondary supply vessel performs at most one closed tour:

$$
\sum_{j \in \mathcal{N}} x_{0 j}^{k} \leq 1, \quad k \in \mathcal{K}_{S}
$$

- Vessels cannot deliver less than the minimum offload amount:

$$
\sum_{j \in \mathcal{N}} \sum_{i \in \mathcal{N}_{f}} y_{j i}^{k}-\sum_{j \in \mathcal{N}} \sum_{i \in \mathcal{N}_{f}} y_{i j}^{k} \geq \sum_{j \in \mathcal{N}} \sum_{i \in \mathcal{N}_{f}} \epsilon_{f}^{k} x_{j i}^{k}, \quad f \in \mathcal{F}, \quad k \in \mathcal{K} .
$$

- Cargo demand requirements:

$$
\sum_{k \in \mathcal{K}} \sum_{j \in \mathcal{N}} \sum_{i \in \mathcal{N}_{f}} y_{j i}^{k}-\sum_{k \in \mathcal{K}} \sum_{j \in \mathcal{N}} \sum_{i \in \mathcal{N}_{f}} y_{i j}^{k}=q_{f}, \quad f \in \mathcal{F}
$$

- Time sequencing constraints (between offshore facilities):

$$
\sum_{i \in \mathcal{N}_{f_{1}}} s_{i}^{k}+\delta_{f_{1}}^{k}+\sum_{i \in \mathcal{N}_{f_{1}}} \sum_{j \in \mathcal{N}_{f_{2}}} \tau_{i j}^{k} x_{i j}^{k} \leq \sum_{i \in \mathcal{N}_{f_{2}}} s_{i}^{k}+M\left\{1-\sum_{i \in \mathcal{N}_{f_{1}}} \sum_{j \in \mathcal{N}_{f_{2}}} x_{i j}^{k}\right\}, \quad f_{1} \in \mathcal{F}, \quad f_{2} \in \mathcal{F}, \quad k \in \mathcal{K} .
$$


- Time sequencing constraints (from the base to an offshore facility - primary vessel):

$$
s_{0}^{k_{P}}+\delta_{0}^{k_{P}}+\sum_{j \in \mathcal{N}_{f}} \tau_{0 j}^{k_{P}} x_{0 j}^{k_{P}}+\sum_{d=1}^{7} \sum_{l=0}^{7} l\left(a_{0}^{d+1}-b_{0}^{d}\right) z_{d l}^{k_{P}} \leq \sum_{j \in \mathcal{N}_{f}} s_{j}^{k_{P}}+M\left\{1-\sum_{j \in \mathcal{N}_{f}} x_{0 j}^{k_{P}}\right\}, \quad f \in \mathcal{F} .
$$

- Time sequencing constraints (from the base to an offshore facility - secondary vessels):

$$
s_{0}^{k}+\delta_{0}^{k}+\sum_{j \in \mathcal{N}_{f}} \tau_{0 j}^{k} x_{0 j}^{k}+\sum_{d=1}^{14} \sum_{l=0}^{14-d} l\left(a_{0}^{d+1}-b_{0}^{d}\right) z_{d l}^{k} \leq \sum_{j \in \mathcal{N}_{f}} s_{j}^{k}+M\left\{1-\sum_{j \in \mathcal{N}_{f}} x_{0 j}^{k}\right\}, \quad f \in \mathcal{F}, \quad k \in \mathcal{K}_{S} .
$$

- Time sequencing constraints (from an offshore facility to the base):

$$
\sum_{j \in \mathcal{N}_{f}} s_{j}^{k}+\delta_{f}^{k}+\sum_{j \in \mathcal{N}_{f}} \tau_{j 0}^{k} x_{j 0}^{k} \leq s_{0}^{k}+t^{k}+M\left\{1-\sum_{j \in \mathcal{N}_{f}} x_{j 0}^{k}\right\}, \quad f \in \mathcal{F}, \quad k \in \mathcal{K} .
$$

- Primary supply vessel must begin base service in the first week:

$$
\sum_{d=1}^{7} \sum_{l=0}^{7} z_{d l}^{k_{P}}=1
$$

- Secondary supply vessels must depart from the base if used:

$$
\sum_{d=1}^{14} \sum_{l=0}^{14-d} z_{d l}^{k}=\sum_{j \in \mathcal{N}} x_{0 j}^{k}, \quad k \in \mathcal{K}_{S}
$$

- Bounds on the base service completion time for the primary vessel:

$$
\sum_{d=1}^{7} \sum_{l=0}^{7} a_{0}^{d+l} z_{d l}^{k_{P}} \leq s_{0}^{k_{P}}+\delta_{0}^{k_{P}}+\sum_{d=1}^{7} \sum_{l=0}^{7} l\left(a_{0}^{d+1}-b_{0}^{d}\right) z_{d l}^{k_{P}} \leq \sum_{d=1}^{7} \sum_{l=0}^{7} b_{0}^{d+l} z_{d l}^{k_{P}} .
$$

- Bounds on the base service completion time for the secondary vessels:

$$
\begin{aligned}
\sum_{d=1}^{14} \sum_{l=0}^{14-d} a_{0}^{d+l} z_{d l}^{k} \leq & s_{0}^{k}+\delta_{0}^{k}+\sum_{d=1}^{14} \sum_{l=0}^{14-d} l\left(a_{0}^{d+1}-b_{0}^{d}\right) z_{d l}^{k} \\
& \leq \sum_{d=1}^{14} \sum_{l=0}^{14-d} b_{0}^{d+l} z_{d l}^{k}+M\left\{1-\sum_{d=1}^{14} \sum_{l=0}^{14-d} z_{d l}^{k}\right\}, \quad k \in \mathcal{K}_{S} .
\end{aligned}
$$

- Primary supply vessel must start before the secondary supply vessels:

$$
M\left\{1-\sum_{d=1}^{14} \sum_{l=0}^{14-d} z_{d l}^{k}\right\}+s_{0}^{k} \geq s_{0}^{k_{P}}, \quad k \in \mathcal{K}_{S}
$$

- Secondary supply vessels must finish before the primary supply vessel:

$$
-M\left\{1-\sum_{d=1}^{14} \sum_{l=0}^{14-d} z_{d l}^{k}\right\}+s_{0}^{k}+t^{k} \leq s_{0}^{k_{P}}+t^{k_{P}}, \quad k \in \mathcal{K}_{S} .
$$

Our decision support model can now be defined as follows: Minimize a linear combination of the objectives (1) and (2) subject to constraints (3)-(26). This problem is a mixed-integer linear programming problem. In the next section, we describe a computational strategy for solving this problem.

Note that $a_{0}^{d+1}$ in constraints (19) and (24) is undefined when $d=14$. This is not a problem, however, because $l=0$ when $d=14$ and thus we always have $l\left(a_{0}^{d+1}-b_{0}^{d}\right)=0$ regardless of the value of $a_{0}^{d+1}$. 


\section{Solution Approach}

Since the mixed-integer linear programming model in Section 2 is usually very challenging to solve for realistic problem instances, we now discuss several methods for simplifying the model. One such method involves using the arrival time windows to determine links that cannot be traversed, and then eliminating the corresponding $x_{i j}^{k}$ and $y_{i j}^{k}$ variables. This is described in the following result.

Theorem 1 Any feasible schedule for the mixed-integer linear programming model in Section 2 satisfies

$$
x_{i j}^{k}=0, \quad y_{i j}^{k}=0, \quad(i, j) \in \mathcal{A}, \quad k \in \mathcal{K}: b_{j}< \begin{cases}a_{i}+\delta_{i}^{k}+\tau_{i j}^{k}, & \text { if } i \in \mathcal{N} \backslash\{0\}, \\ a_{0}^{1}+\delta_{0}^{k}+\tau_{0 j}^{k}, & \text { if } i=0 .\end{cases}
$$

Proof The earliest time that vessel $k$ can reach node $j$ from node $i$ is $a_{i}+\delta_{i}^{k}+\tau_{i j}^{k}$ for $i \in \mathcal{N} \backslash\{0\}$ and $a_{0}^{1}+\delta_{0}^{k}+\tau_{0 j}^{k}$ for $i=0$. Clearly, if $b_{j}$ is less than the earliest possible arrival time at node $j$, then vessel $k$ cannot traverse link $(i, j)$, and the corresponding $x_{i j}^{k}$ and $y_{i j}^{k}$ variables must be zero.

Now, define

$$
\theta^{k}=\left\lceil\frac{\delta_{0}^{k}}{b_{0}^{1}-a_{0}^{1}}\right\rceil
$$

The base service for vessel $k$ must take place over at least $\theta^{k}$ distinct days. For example, if $\delta_{0}^{k}=17$ and the base is open for 12 hours each day, then vessel $k$ 's service at the base must $\operatorname{span} \theta^{k}=2$ days. Our next result shows that $z_{d l}^{k}$ for $l \notin\left\{\theta^{k}-1, \theta^{k}\right\}$ can be eliminated from the model.

Theorem 2 Any feasible schedule for the mixed-integer linear programming model in Section 2 satisfies

$$
z_{d l}^{k}=0, \quad d \in\{1, \ldots, 14\}, \quad l \notin\left\{\theta^{k}-1, \theta^{k}\right\}, \quad k \in \mathcal{K}
$$

Proof Suppose, to the contrary, that there exists a feasible schedule with $z_{d l}^{k}=1$ for some $l \leq \theta^{k}-2$ or $l \geq \theta^{k}+1$. If $l \leq \theta^{k}-2$, then vessel $k$ spends at most $l+1 \leq \theta^{k}-1$ full days in service at the base. Hence, the number of working hours that vessel $k$ spends in service at the base cannot exceed

$$
\left(\theta^{k}-1\right)\left(b_{0}^{1}-a_{0}^{1}\right)=\left(\left\lceil\frac{\delta_{0}^{k}}{b_{0}^{1}-a_{0}^{1}}\right\rceil-1\right)\left(b_{0}^{1}-a_{0}^{1}\right)<\frac{\delta_{0}^{k}}{b_{0}^{1}-a_{0}^{1}}\left(b_{0}^{1}-a_{0}^{1}\right)=\delta_{0}^{k} .
$$

This is a contradiction as vessel $k$ must spend at least $\delta_{0}^{k}$ open hours at the base to complete the service.

Now, if $l \geq \theta^{k}+1$, then vessel $k$ spends at least $l-1 \geq \theta^{k}$ full days in service at the base. Thus, since the base service takes $\delta_{0}^{k}$ working hours,

$$
\delta_{0}^{k} \geq(l-1)\left(b_{0}^{1}-a_{0}^{1}\right) \geq \theta^{k}\left(b_{0}^{1}-a_{0}^{1}\right)=\left\lceil\frac{\delta_{0}^{k}}{b_{0}^{1}-a_{0}^{1}}\right\rceil\left(b_{0}^{1}-a_{0}^{1}\right) \geq \delta_{0}^{k} .
$$


Hence,

$$
\delta_{0}^{k}=(l-1)\left(b_{0}^{1}-a_{0}^{1}\right) .
$$

It follows that up to the end of day $d+l-1$, the number of working hours spent by vessel $k$ in service at the base is

$$
b_{0}^{d}-s_{0}^{k}+(l-1)\left(b_{0}^{1}-a_{0}^{1}\right)=b_{0}^{d}-s_{0}^{k}+\delta_{0}^{k} \geq \delta_{0}^{k} .
$$

But this implies that vessel $k$ 's service is complete by the end of day $d+l-1$, contradicting $z_{d l}^{k}=1$.

Theorems 1 and 2 can be used to eliminate many of the decision variables and thereby reduce the size and complexity of the model. In addition, we can eliminate variables that pertain to travel between nodes corresponding to the same facility:

$$
x_{i j}^{k}=0, \quad y_{i j}^{k}=0, \quad i \in \mathcal{N}_{f}, \quad j \in \mathcal{N}_{f}, \quad f \in \mathcal{F}, \quad k \in \mathcal{K} .
$$

Moreover, since returning unused cargo to the base is clearly non-optimal, we can eliminate the cargo flow variables for arcs going back to the base:

$$
y_{i 0}^{k}=0, \quad i \in \mathcal{N}_{f}, \quad f \in \mathcal{F}, \quad k \in \mathcal{K} .
$$

In our numerical experiments (see Section 4), the variable assignments described in Theorems 1 and 2 and equations (27) and (28) typically result in dimension reductions of at least 50\%. Despite this, the reduced model is still difficult to solve in large dimensions. Thus, we now describe a heuristic method for generating an initial feasible schedule, which can be used to accelerate the convergence of mixed-integer programming solvers such as CPLEX.

The heuristic method involves solving two subproblems. The first subproblem, called Subproblem $\mathrm{A}\left(d_{P}\right)$, is formed by eliminating the secondary supply vessels and cargo demands and restricting the primary vessel's start day to day $d_{P}$. The second subproblem, called Subproblem $\mathrm{B}\left(d_{P}, d_{S}\right)$, is formed by fixing the primary vessel's tour to the optimal solution of Subproblem $\mathrm{A}\left(d_{P}\right)$, restricting the secondary vessels' start day to day $d_{S}$, and including the cargo flow variables for all vessels.

More specifically, Subproblem $\mathrm{A}\left(d_{P}\right)$ is constructed by defining

- $\emptyset \rightarrow \mathcal{K}_{S}$

- $0 \rightarrow q_{f}$ for each $f \in \mathcal{F}$;

- $0 \rightarrow y_{i j}^{k_{P}}$ for each $(i, j) \in \mathcal{A}$;

- $0 \rightarrow z_{d l}^{k_{P}}$ for each $d \neq d_{P}$ and $l \in\{0, \ldots, 14-d\}$; and

- $0 \rightarrow x_{0 j}^{k_{P}}$ for each $j \in \mathcal{N}: b_{j}<a_{0}^{d_{P}}+\delta_{0}^{k_{P}}+\tau_{0 j}^{k_{P}}$. 


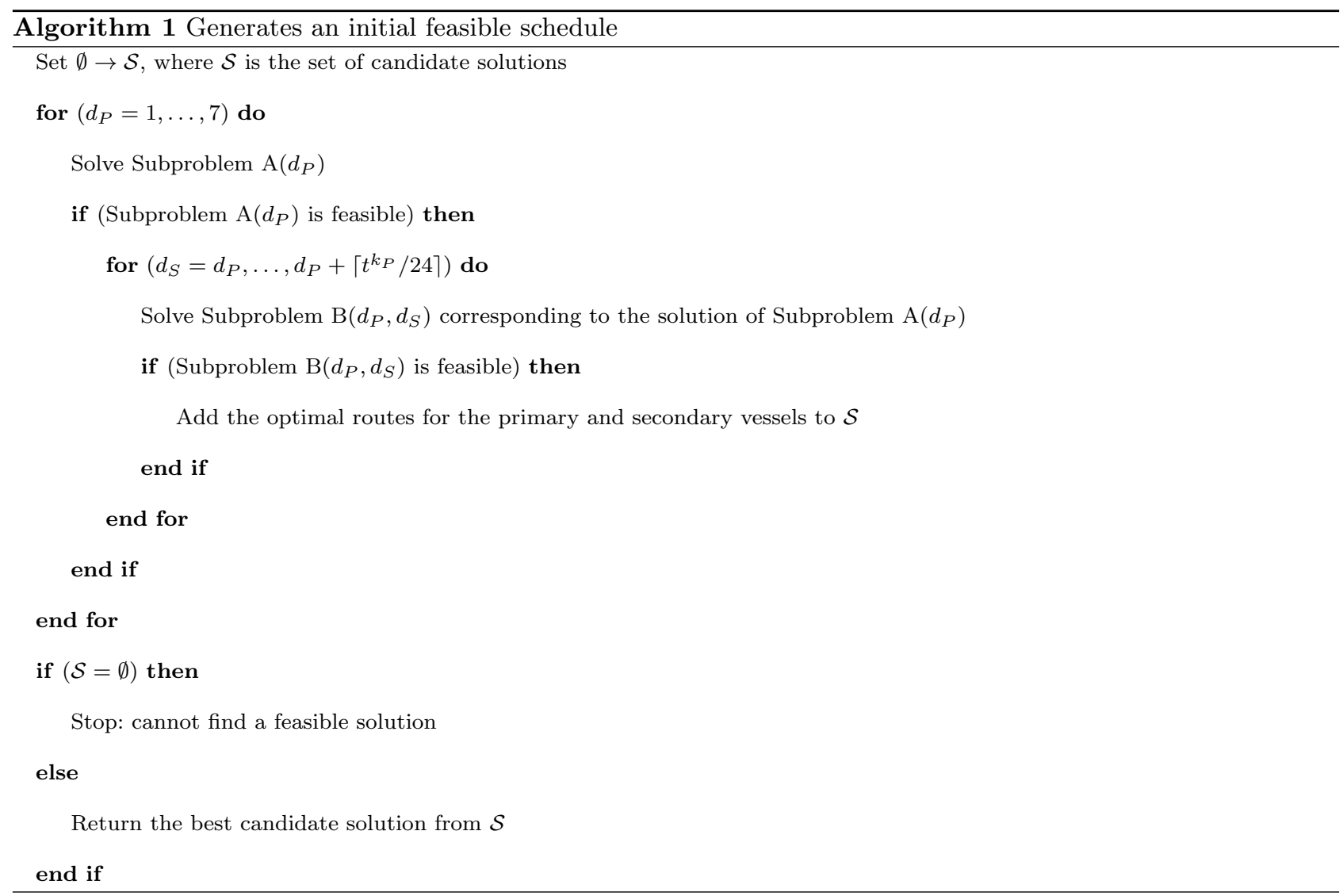

Subproblem $\mathrm{B}\left(d_{P}, d_{S}\right)$ is constructed by fixing variables $\left(x_{i j}^{k_{P}}, z_{d_{P} l}^{k_{P}}, s_{i}^{k_{P}}, s_{0}^{k_{P}}, t^{k_{P}}\right)$ to the optimal solution of Subproblem $\mathrm{A}\left(d_{P}\right)$, and then defining

- $0 \rightarrow z_{d l}^{k_{P}}$ for each $d \neq d_{P}$ and $l \in\{0, \ldots, 14-d\}$;

- $0 \rightarrow z_{d l}^{k}$ for each $d \neq d_{S}, l \in\{0, \ldots, 14-d\}$, and $k \in \mathcal{K}_{S}$;

- $0 \rightarrow x_{0 j}^{k}$ for each $j \in \mathcal{N}$ and $k \in \mathcal{K}_{S}: b_{j}<a_{0}^{d_{S}}+\delta_{0}^{k}+\tau_{0 j}^{k}$; and

- $0 \rightarrow y_{0 j}^{k}$ for each $j \in \mathcal{N}$ and $k \in \mathcal{K}_{S}: b_{j}<a_{0}^{d_{S}}+\delta_{0}^{k}+\tau_{0 j}^{k}$.

The pseudocode for our heuristic algorithm is detailed in Algorithm 1. The algorithm repeatedly solves Subproblems $\mathrm{A}\left(d_{P}\right)$ and $\mathrm{B}\left(d_{P}, d_{S}\right)$, where Subproblem $\mathrm{A}\left(d_{P}\right)$ involves optimizing the primary vessel's tour (for start day $\left.d_{P}\right)$ while ignoring the secondary vessels, and Subproblem $\mathrm{B}\left(d_{P}, d_{S}\right)$ involves optimizing the secondary vessels' tours (for start day $d_{S}$ ) while the primary vessel's tour is fixed to the optimal solution of Subproblem $\mathrm{A}\left(d_{P}\right)$. The possible values for $d_{P}$ and $d_{S}$ are $d_{P}=1, \ldots, 7$ and $d_{S}=d_{P}, \ldots, d_{P}+\left\lceil t^{k_{P}} / 24\right\rceil$ (recall that the tours for the secondary vessels must take place within the primary vessel's tour window). The algorithm solves Subproblems $\mathrm{A}\left(d_{P}\right)$ and $\mathrm{B}\left(d_{P}, d_{S}\right)$ for each of these values and returns the best candidate solution obtained. If Algorithm 1 terminates with a candidate solution, then this solution is certainly feasible for the overall problem, but not nec- 
essarily optimal. The reason is that Algorithm 1 optimizes the primary vessel first in a greedy fashion before optimizing the secondary vessels. This sequence may not yield an overall optimal result.

If Subproblem $\mathrm{A}\left(d_{P}\right)$ is infeasible for each $d_{P}=1, \ldots, 7$, then the overall problem must be infeasible; the time window constraints on the primary vessel are too restrictive. Alternatively, if Subproblem $\mathrm{A}\left(d_{P}\right)$ is feasible for at least one $d_{P}$, but the corresponding Subproblems $\mathrm{B}\left(d_{P}, d_{S}\right)$ are all infeasible, then the overall problem is also infeasible. This is because any feasible schedule for the overall problem can be converted into a feasible schedule of Subproblem $\mathrm{B}\left(d_{P}, d_{S}\right)$ by choosing $d_{P}$ to be the start day of the primary vessel, and $d_{S}$ to be the minimum start day over all secondary vessels. Secondary vessels starting on a later day can be assumed to start on day $d_{S}$ by adding a sufficiently long waiting period after the initial base service. Although the model assumes that base servicing is conducted first, in reality the base service can be done at any time while the vessel is in port.

Fixing the primary vessel's tour in Subproblem $\mathrm{B}\left(d_{P}, d_{S}\right)$ greatly simplifies the model, and thus it may be possible to relax Subproblem B $\left(d_{P}, d_{S}\right)$ by eliminating the requirement that all secondary vessels start on day $d_{S}$, instead allowing any start day within the primary vessel's tour window. This will potentially give a better initial solution, although for the case study considered in this paper, Algorithm 1 already yields solutions that are very close to optimal (see the results in the next section).

\section{Numerical Results}

We consider eight Woodside-operated offshore oil and gas facilities in the Indian Ocean off the coast of Western Australia: Angel, Goodwyn A, Nganhurra, Ngujima-Yin, North Rankin A, North Rankin B, Okha, and Pluto. The locations of these facilities are shown in Figure 1, where GWA denotes Goodwyn A, NGA denotes Nganhurra, NY denotes Ngujima-Yin, and NRC denotes North Rankin A and B (these two platforms are very close and are actually connected; thus, they are represented by a single point in Figure 1).

Our goal in this case study is to design an optimal vessel schedule for servicing the offshore facilities in Figure 1 using one platform supply vessel (PSV) and one off-take support vessel (OSV). The PSV is used solely for cargo delivery, while the OSV is used for both cargo delivery and oil off-takes (with off-takes being the OSV's primary function). Thus, since we focus on cargo operations in this case study, the PSV is the primary vessel and the OSV is the secondary vessel. The base for both vessels is King Bay supply base (KBSB) located near Karratha.

The deck-space capacity, speed, and fuel consumption rate for each vessel are given in Table 1. The distances between facilities are given in Table 2. Since both vessels travel at a speed of 10 knots, the travel times can be 


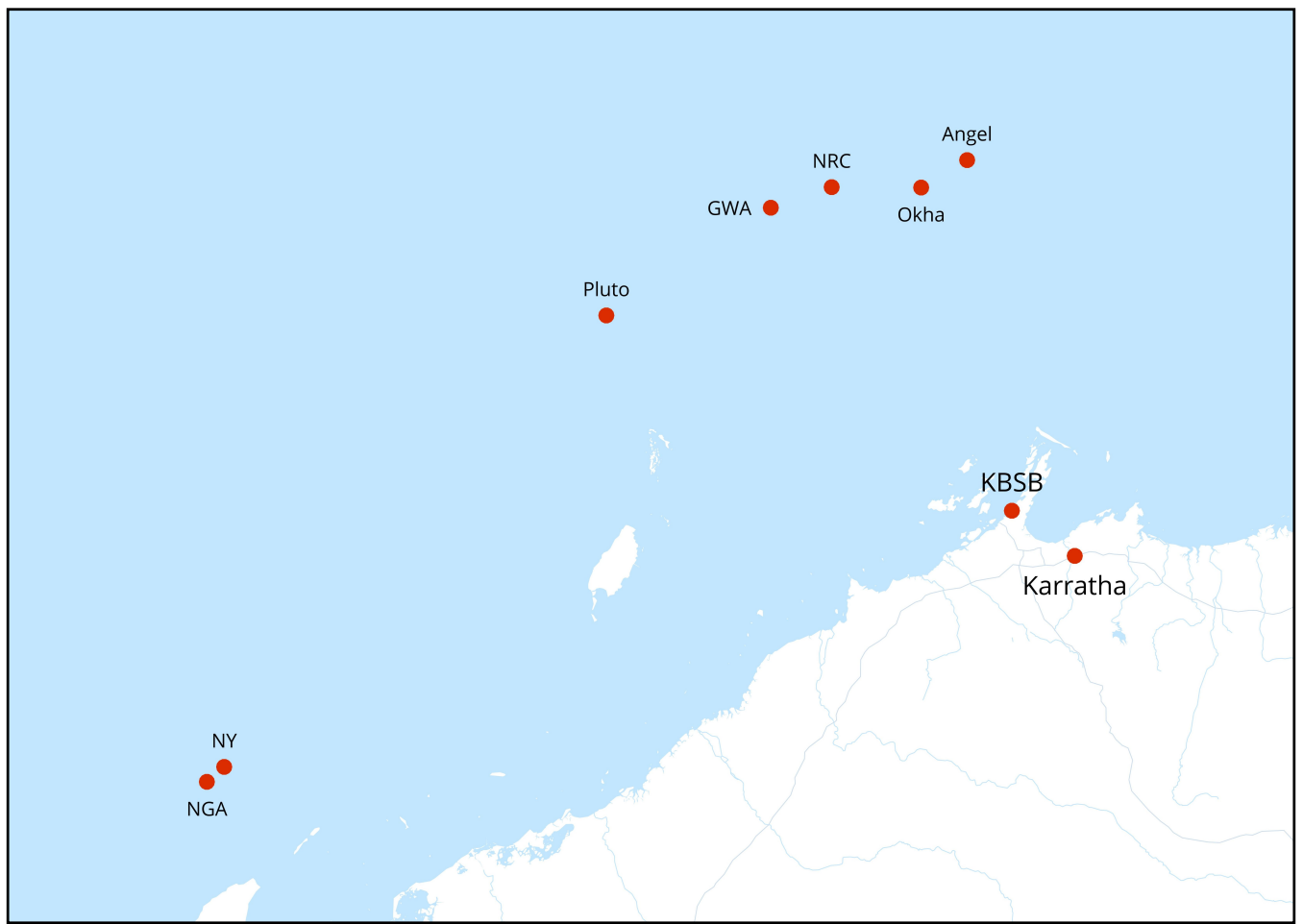

Fig. 1 Locations of the oil and gas facilities in the case study in Section 4.

\begin{tabular}{lccc}
\hline Vessel & Capacity $\left(\mathrm{m}^{2}\right)$ & Speed (Knots) & Fuel Consumption (L/NM) \\
\hline PSV & 850.0 & 10 & 54 \\
OSV & 212.5 & 10 & 40 \\
\hline
\end{tabular}

Table 1 Deck-space capacity, speed, and fuel consumption rate for each vessel.

obtained by dividing the numbers in Table 2 by 10 . North Rankin A and B, although connected by a bridge, still require separate cargo deliveries. The time taken for a vessel to manoeuvre between North Rankin A and B is 30 minutes. Moreover, service time at the base is 21 hours for the PSV and 10.5 hours for the OSV.

In real operations, the OSV is normally reserved for off-take support, and is only used for cargo delivery when the PSV cannot fulfill all cargo demands. Therefore, we choose the following cost function to penalize OSV usage in addition to trip duration and fuel consumption:

$$
\text { Total Cost }=t^{k_{P}}+t^{k_{S}}+\sum_{(i, j) \in \mathcal{A}} 540 \tau_{i j}^{k_{P}} x_{i j}^{k_{P}}+\sum_{(i, j) \in \mathcal{A}} 400 \tau_{i j}^{k_{S}} x_{i j}^{k_{S}}-\sum_{f \in \mathcal{F}} \sum_{j \in \mathcal{N}_{f}} y_{0 j}^{k_{P}}+\sum_{f \in \mathcal{F}} \sum_{j \in \mathcal{N}_{f}} y_{0 j}^{k_{S}},
$$

where $k_{P}$ refers to the PSV, $k_{S}$ refers to the OSV, $\tau_{i j}^{k_{P}}$ is the PSV's travel time for link $(i, j)$, and $\tau_{i j}^{k_{S}}$ is the OSV's travel time for link $(i, j)$. Thus, in the framework of the model in Section 2, the fixed and variable costs are $\alpha_{i j}^{k_{P}}=540 \tau_{i j}^{k_{P}}, \alpha_{i j}^{k_{S}}=400 \tau_{i j}^{k_{S}}, \beta_{0 j}^{k_{P}}=-1, \beta_{0 j}^{k_{S}}=1$, and $\beta_{i j}^{k_{P}}=\beta_{i j}^{k_{S}}=0$ for $i \neq 0$. Here, the fixed cost coefficients 


\begin{tabular}{ccccccccc}
\hline & KBSB & Angel & GWA & NGA & NY & NRC & Okha & Pluto \\
KBSB & - & 68.4 & 78.4 & 180.0 & 175.0 & 75.0 & 65.0 & 95.9 \\
Angel & 68.4 & - & 38.4 & 188.4 & 181.7 & 27.5 & 10.0 & 75.0 \\
GWA & 78.4 & 38.4 & - & 155.0 & 155.9 & 12.5 & 30.0 & 38.4 \\
NGA & 180.0 & 188.4 & 155.0 & - & 5.0 & 165.0 & 170.0 & 117.5 \\
NY & 175.0 & 181.7 & 155.9 & 5.0 & - & 160.0 & 165.0 & 112.5 \\
NRC & 75.0 & 27.5 & 12.5 & 165.0 & 160.0 & - & 18.4 & 50.0 \\
Okha & 65.0 & 10.0 & 30.0 & 170.0 & 165.0 & 18.4 & - & 65.0 \\
Pluto & 95.9 & 75.0 & 38.4 & 117.5 & 112.5 & 50.0 & 65.0 & - \\
\hline
\end{tabular}

Table 2 Distances (in nautical miles) between facilities.

measure fuel consumption while the variable cost coefficients are penalty factors designed to maximize PSV use for cargo deliveries.

The minimum, average, and maximum cargo demand per week for each offshore facility are given in Table 3. The service duration at each offshore facility is $\delta_{i}^{k}=6$ hours and the minimum cargo offload is $20.5 \mathrm{~m}^{2}$ (half of the smallest demand in Table 3). The base is open from 6 am to $6 \mathrm{pm}$ every day including weekends, i.e., $\left[a_{0}^{d}, b_{0}^{d}\right]=[24 d-18,24 d-6]$ for each $d \in\{1, \ldots, 14\}$. We assume that Goodwyn A and the North Rankin complex operate continuously. The other facilities are open from 6 am to $6 \mathrm{pm}$ every day including weekends.

A problem scenario is defined by the helicopter schedule and the cargo demands and diesel requirements at the offshore facilities. The helicopter schedule defines the set of time windows for each offshore facility (vessels cannot arrive while a facility is being prepared for helicopter arrival). Table 4 shows the helicopter preparation intervals provided by Woodside. Vessels cannot arrive during these intervals, as all personnel are required to prepare for helicopter arrival.

In addition to the helicopter schedule, diesel deliveries further constrain the service time windows at the 24hour facilities Goodwyn A and North Rankin A and B. Specifically, we assume that diesel deliveries cannot be performed at night. Thus, when diesel delivery is required, service visits at Goodwyn A and North Rankin A and B must be restricted to daylight hours.

We consider two problem sets: the first problem set involves all eight offshore facilities with no diesel shipments; the second problem set involves all facilities except Angel, with diesel required at Goodwyn A. These were two of the scenarios of interest to Woodside. 


\begin{tabular}{cccc}
\hline & \multicolumn{3}{c}{ Weekly Cargo Demand $\left(\mathrm{m}^{2}\right)$} \\
\cline { 2 - 4 } Facility & Minimum & Average & Maximum \\
\hline Angel & 20 & 45 & 67 \\
Goodwyn A & 133 & 287 & 463 \\
Nganhurra & 106 & 133 & 188 \\
Ngujima-Yin & 74 & 127 & 196 \\
North Rankin A & 77 & 160 & 284 \\
North Rankin B & 65 & 124 & 262 \\
Okha & 43 & 102 & 198 \\
Pluto & 6 & 41 & 141 \\
\hline
\end{tabular}

Table 3 Weekly cargo demand statistics for the offshore facilities.

\begin{tabular}{cccccc}
\hline & Monday & Tuesday & Wednesday & Thursday & Friday \\
GWA & $10: 25 \mathrm{am}-11: 20 \mathrm{am}$ & $12: 35 \mathrm{pm}-1: 40 \mathrm{pm}$ & $8: 35 \mathrm{am}-9: 30 \mathrm{am}$ & $10: 25 \mathrm{am}-11: 20 \mathrm{am}$ & $10: 25 \mathrm{am}-11: 20 \mathrm{am}$ \\
NGA & $8: 00 \mathrm{am}-8: 50 \mathrm{am}$ & - & $8: 00 \mathrm{am}-8: 50 \mathrm{am}$ & - & - \\
NY & $9: 30 \mathrm{am}-10: 55 \mathrm{am}$ & - & $9: 30 \mathrm{am}-10: 55 \mathrm{am}$ & - & $8: 20 \mathrm{am}-9: 05 \mathrm{am}$ \\
NRC & $8: 35 \mathrm{am}-9: 15 \mathrm{am}$ & $8: 35 \mathrm{am}-9: 30 \mathrm{am}$ & $10: 25 \mathrm{am}-11: 20 \mathrm{am}$ & $8: 35 \mathrm{am}-9: 15 \mathrm{am}$ & $8: 35 \mathrm{am}-9: 15 \mathrm{am}$ \\
Okha & - & $10: 25 \mathrm{am}-11: 20 \mathrm{am}$ & - & $8: 50 \mathrm{am}-9: 30 \mathrm{am}$ & - \\
\hline
\end{tabular}

Table 4 Helicopter preparation intervals for the offshore facilities.

\subsection{Eight Facilities with No Diesel}

We first considered the scheduling problem with all eight facilities, no diesel shipments, and the average cargo demands in Table 3. Applying the optimization software CPLEX (embedded in the AIMMS modelling platform) to the full integer programming model took 5.12 hours to obtain an optimal solution. To simplify the model, we applied the variable reduction techniques discussed in Section 3, which reduced the optimization model from 21,800 binary variables, 21,843 continuous variables, and 22,455 constraints to 9,484 binary variables, 9,653 continuous variables, and 10,265 constraints. We then solved the reduced problem using a combination of CPLEX and the heuristic method in Section 3 (for generating an initial feasible schedule). This took 24.71 minutes, far less than the time taken to solve the full model.

For this problem instance, the OSV is required in addition to the PSV to satisfy the demand requirements; the optimal visit sequences are illustrated in Figure 2. The total fuel consumption is $32,082 \mathrm{~L}$ and the journey 


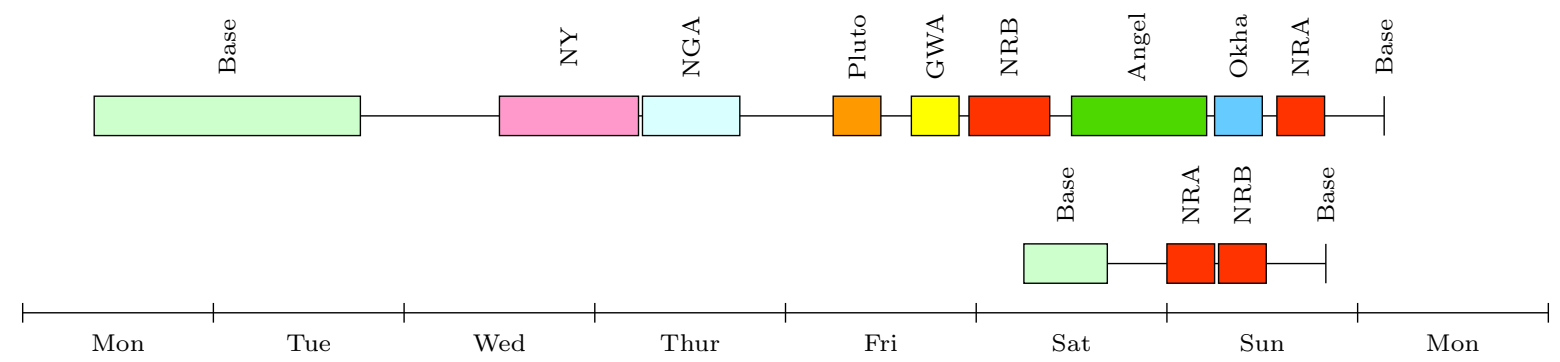

Fig. 2 Optimal vessel routes for eight facilities with average demand requirements and no diesel: the top route is the PSV's route; the bottom route is the OSV's route.

durations are 162.34 hours for the PSV and 38.00 hours for the OSV. Moreover, the PSV's deck-space utilization is $100 \%$ and the OSV's deck-space utilization is $80 \%$. As expected, the PSV is fully utilized at the optimal solution (recall that the OSV is only used when the PSV is full).

We next generated 20 additional problem scenarios by changing the demand at each facility to random values generated by a uniform distribution between the minimum and maximum demands (see Table 3), keeping all other parameters the same. Thus, each of these 20 problem scenarios involves eight facilities and no diesel shipments, but the demand requirements vary. Note that the average values in Table 3 are the average demands from the historical data, not the means of the uniform distributions used to generate the problem scenarios.

We solved each scenario using CPLEX and the heuristic method in Section 3. All scenarios were solved to optimality except scenarios 1 and 8 , which were terminated after 2 hours of computation with optimality gaps of $1.98 \%$ and $4.38 \%$, respectively. Interestingly, the final PSV route for each scenario is the same as the PSV route shown in Figure 2, but the OSV route is different. This indicates that the PSV route mainly depends on the helicopter schedule, which is fixed in the 20 problem scenarios. The OSV route, on the other hand, depends on the demand requirements and is different for each scenario. See Table 5 for a summary of the results in these problem scenarios. The "Improvement" column in Table 5 refers to the difference in objective values for the initial and final schedules as a percentage of the final objective value. The values in the improvement column are very small, indicating that the heuristic method generates near-optimal solutions and only a small improvement is obtained by running CPLEX on the full model. 


\begin{tabular}{|c|c|c|c|c|c|}
\hline \multirow[b]{2}{*}{ Scenario } & \multirow[b]{2}{*}{ Total Demand $\left(\mathrm{m}^{2}\right)$} & \multirow[b]{2}{*}{ Improvement } & \multicolumn{2}{|l|}{ OSV Usage } & \multirow[b]{2}{*}{ Fuel Consumption (L) } \\
\hline & & & Trip Duration (Hours) & Deck-space Utilization & \\
\hline 1 & 1048.0 & $0.0382 \%$ & 31.5 & $93.2 \%$ & 6000.0 \\
\hline 2 & 801.4 & $0.0000 \%$ & 0.0 & $0.0 \%$ & 0.0 \\
\hline 3 & 977.7 & $0.0445 \%$ & 59.0 & $60.1 \%$ & 5736.0 \\
\hline 4 & 1024.8 & $0.0382 \%$ & 31.5 & $82.3 \%$ & 6000.0 \\
\hline 5 & 1027.4 & $0.0382 \%$ & 31.5 & $83.5 \%$ & 6000.0 \\
\hline 6 & 825.3 & $0.0000 \%$ & 0.0 & $0.0 \%$ & 0.0 \\
\hline 7 & 980.8 & $0.0442 \%$ & 35.0 & $61.6 \%$ & 5200.0 \\
\hline 8 & 1056.9 & $0.0379 \%$ & 38.0 & $97.3 \%$ & 6200.0 \\
\hline 9 & 902.0 & $0.0446 \%$ & 59.0 & $24.5 \%$ & 5736.0 \\
\hline 10 & 999.2 & $0.0382 \%$ & 31.5 & $70.2 \%$ & 6000.0 \\
\hline 11 & 987.2 & $0.0380 \%$ & 38.0 & $64.6 \%$ & 6200.0 \\
\hline 12 & 1040.5 & $0.0440 \%$ & 59.0 & $89.6 \%$ & 5736.0 \\
\hline 13 & 1007.4 & $0.0382 \%$ & 31.5 & $74.1 \%$ & 6000.0 \\
\hline 14 & 780.5 & $0.0000 \%$ & 0.0 & $0.0 \%$ & 0.0 \\
\hline 15 & 1033.6 & $0.0380 \%$ & 38.0 & $86.4 \%$ & 6200.0 \\
\hline 16 & 967.7 & $0.0383 \%$ & 31.5 & $55.4 \%$ & 6000.0 \\
\hline 17 & 1016.4 & $0.0382 \%$ & 31.5 & $78.3 \%$ & 6000.0 \\
\hline 18 & 1029.1 & $0.0382 \%$ & 31.5 & $84.3 \%$ & 6000.0 \\
\hline 19 & 1044.8 & $0.0379 \%$ & 38.0 & $91.7 \%$ & 6200.0 \\
\hline 20 & 1021.5 & $0.0444 \%$ & 59.0 & $80.7 \%$ & 5736.0 \\
\hline
\end{tabular}

Table 5 Results for the 20 randomly-generated problem scenarios in Section 4.1.

\subsection{Seven Facilities (Angel Omitted) with Diesel at Goodwyn A}

Unlike the other facilities, Angel and Pluto are normally unmanned and do not require regular cargo deliveries. This is why the average demands for Angel and Pluto in Table 3 are significantly less than those for the other facilities.

We considered a scheduling scenario in which Pluto, but not Angel, requires cargo delivery, and diesel is required at Goodwyn A. The cargo demands for this scenario were chosen as the average demands given in Table 3. Using our variable reduction techniques (see Section 3), the optimization model was reduced from 13,946 binary variables, 13,947 continuous variables, and 14,438 constraints to 5,849 binary variables, 5,976 continuous variables, and 6,467 constraints. Solving the problem using a combination of CPLEX and the heuristic method in Section 3 yields the optimal routes shown in Figure 3. The total fuel consumption is 29,587 L and the optimal route durations are 156.09 hours for the PSV and 31.50 hours for the OSV. Moreover, the deck-space utilization 


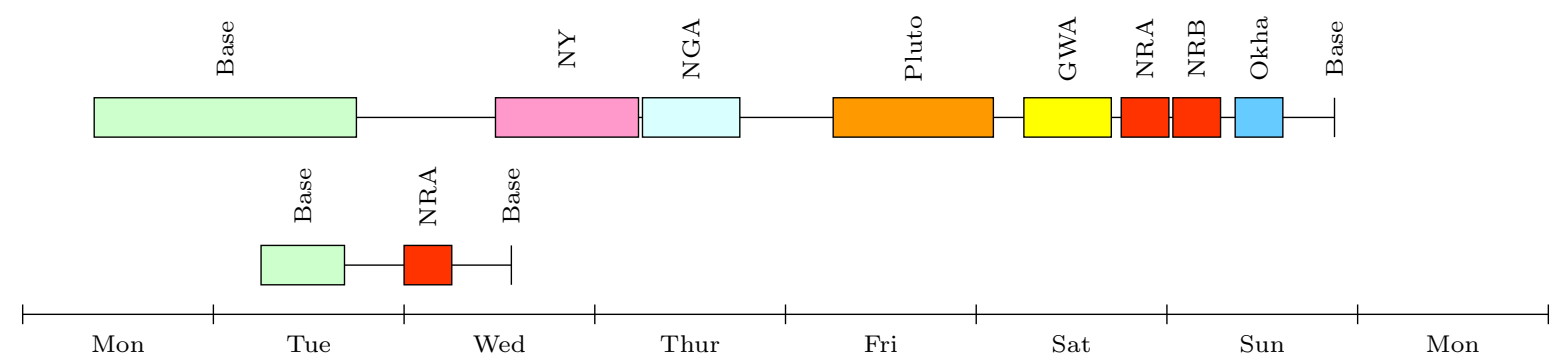

Fig. 3 Optimal vessel routes for seven facilities (Angel omitted) with average demand requirements and diesel at GWA: the top route is the PSV's route; the bottom route is the OSV's route.

of the PSV is $100 \%$ and the deck-space utilization of the OSV is $58 \%$. For this example, solving the full integer programming model took 42.9 seconds; the method described in Section 3 took 23.9 seconds.

As in Section 4.1, we generated 20 additional problem scenarios by randomly choosing the cargo demand at each facility between the minimum and maximum values in Table 3. Solving these scenarios reveals that there is no change in the optimal PSV route, but the optimal OSV route does change depending on the demand requirements. All problem scenarios were solved to optimality and Table 6 gives the results. The "Improvement" column in Table 6 has the same meaning as in Table 5. As with the scenarios in Section 4.1, the results in Table 6 show that solutions obtained by the heuristic method are very close to optimal.

\section{Conclusion}

This paper has described a real example of where mixed-integer linear programming techniques have been applied to aid strategic decision-making in the oil and gas industry. The scheduling problem we considered, as with most realworld scheduling problems, is computationally challenging. Indeed, in our experience, CPLEX struggles to solve the full (unsimplified) problem unless a good starting point is provided. The heuristic decomposition procedure in Section 3 was designed for this purpose. The optimization model described in this paper has been implemented as a basic scheduling tool, and further improvements are planned to enable dynamic schedule updates "on the fly" in response to unforeseen events such as cyclones and equipment breakdowns. Our current model determines the optimal vessel routes given fixed helicopter arrival times. Clearly, simultaneously optimizing the vessel and helicopter schedules will yield better results, although this was outside the project brief defined by Woodside. We plan to investigate the combined vessel-helicopter scheduling problem in future work. We also plan to extend the model to allow for cargo delivery interruptions at the offshore facilities during facility closure or helicopter preparation. Our current model only allows service interruptions at the base. 


\begin{tabular}{|c|c|c|c|c|c|}
\hline \multirow[b]{2}{*}{ Scenario } & \multirow[b]{2}{*}{ Total Demand $\left(\mathrm{m}^{2}\right)$} & \multirow[b]{2}{*}{ Improvement } & \multicolumn{2}{|l|}{ OSV Usage } & \multirow[b]{2}{*}{ Fuel Consumption (L) } \\
\hline & & & Trip Duration (Hours) & Deck-space Utilization & \\
\hline 1 & 962.0 & $0.0410 \%$ & 38.0 & $52.7 \%$ & 6200.0 \\
\hline 2 & 683.2 & $0.0000 \%$ & 0.0 & $0.0 \%$ & 0.0 \\
\hline 3 & 1046.6 & $0.0409 \%$ & 38.0 & $92.5 \%$ & 6200.0 \\
\hline 4 & 1016.8 & $0.0412 \%$ & 31.5 & $78.5 \%$ & 6000.0 \\
\hline 5 & 835.9 & $0.0000 \%$ & 0.0 & $0.0 \%$ & 0.0 \\
\hline 6 & 906.2 & $0.0414 \%$ & 31.5 & $26.5 \%$ & 6000.0 \\
\hline 7 & 1031.8 & $0.0412 \%$ & 31.5 & $85.6 \%$ & 6000.0 \\
\hline 8 & 919.0 & $0.0479 \%$ & 35.0 & $32.5 \%$ & 5200.0 \\
\hline 9 & 955.8 & $0.0478 \%$ & 35.0 & $49.8 \%$ & 5200.0 \\
\hline 10 & 1049.7 & $0.0412 \%$ & 31.5 & $94.0 \%$ & 6000.0 \\
\hline 11 & 805.8 & $0.0000 \%$ & 0.0 & $0.0 \%$ & 0.0 \\
\hline 12 & 886.6 & $0.0479 \%$ & 35.0 & $18.5 \%$ & 5200.0 \\
\hline 13 & 976.0 & $0.0413 \%$ & 31.5 & $59.3 \%$ & 6000.0 \\
\hline 14 & 1039.2 & $0.0412 \%$ & 31.5 & $89.0 \%$ & 6000.0 \\
\hline 15 & 916.3 & $0.0414 \%$ & 31.5 & $31.2 \%$ & 6000.0 \\
\hline 16 & 819.9 & $0.0000 \%$ & 0.0 & $0.0 \%$ & 0.0 \\
\hline 17 & 880.6 & $0.0479 \%$ & 35.0 & $14.4 \%$ & 5200.0 \\
\hline 18 & 979.6 & $0.0410 \%$ & 38.0 & $61.0 \%$ & 6200.0 \\
\hline 19 & 988.7 & $0.0410 \%$ & 38.0 & $65.3 \%$ & 6200.0 \\
\hline 20 & 941.4 & $0.0414 \%$ & 31.5 & $43.0 \%$ & 6000.0 \\
\hline
\end{tabular}

Table 6 Results for the 20 randomly-generated problem scenarios in Section 4.2.

\section{Acknowledgements}

This research was funded by Woodside Energy Ltd under the R2D3 Energy Research Alliance Framework, and by the Australian Research Council through the ARC Linkage Scheme. The authors especially thank Rob Duncanson from Woodside for his support of this paper. The authors also thank AIMMS for providing access to the AIMMS software platform.

\section{Disclaimer}

The views expressed in this paper are the authors' personal views and are not attributable to Woodside Energy Ltd.

The paper is intended to be a general discussion and does not constitute advice. Woodside disclaims all liability for 
any loss or damage incurred by any person or organization as a result of using, acting on, relying on, or disclosing any of the information contained in this paper or otherwise in connection with this paper.

\section{References}

1. Belfiore, P., Yoshizaki, H.T.: Heuristic methods for the fleet size and mix vehicle routing problem with time windows and split deliveries. Computers \& Industrial Engineering 64(2), 589-601 (2013)

2. Cordeau, J.F., Laporte, G., Mercier, A.: Improved tabu search algorithm for the handling of route duration constraints in vehicle routing problems with time windows. Journal of the Operational Research Society 55(5), 542-546 (2004)

3. Gulczynski, D., Golden, B., Wasil, E.: The split delivery vehicle routing problem with minimum delivery amounts. Transportation Research Part E: Logistics and Transportation Review 46(5), 612-626 (2010)

4. Koç, Ç., Bektaş, T., Jabali, O., Laporte, G.: Thirty years of heterogeneous vehicle routing. European Journal of Operational Research 249(1), 1-21 (2016)

5. Mancini, S.: A real-life multi depot multi period vehicle routing problem with a heterogeneous fleet: Formulation and adaptive large neighborhood search based matheuristic. Transportation Research Part C: Emerging Technologies (2016)

6. Soonpracha, K., Mungwattana, A., Janssens, G.K., Manisri, T.: Heterogeneous vrp review and conceptual framework. In: Proceedings of the International Multiconference of Engineers and Computer Scientists, Hong Kong (2014)

7. Tavakkoli-Moghaddam, R., Safaei, N., Kah, M., Rabbani, M.: A new capacitated vehicle routing problem with split service for minimizing fleet cost by simulated annealing. Journal of the Franklin Institute 344(5), 406-425 (2007) 